\title{
Prof. Dr. rer. nat. Heinrich Strecker in memoriam
}

\section{Rolf Wiegert}

Online publiziert: 22. Mai 2014

(C) Springer-Verlag Berlin Heidelberg 2014

Geboren wurde Heinrich Strecker am 13. Sept. 1922 in Coburg als Sohn von Studienprofessor Heinrich Strecker sen. und dessen Ehefrau Else Strecker. Der Vater wirkte als Lehrer am berühmten Gymnasium Casimirianum in Coburg, einer Lehranstalt, an welcher später auch sein Sohn das Abitur ablegte. Als väterliches Erbe wurde dem Sohn für den angestrebten akademischen Lebensweg die Neigung und die Fähigkeit zum Studium der Mathematik und Statistik mitgegeben.

Nach der Reifeprüfung 1941 begann Heinrich Strecker mit dem Studium der Mathematik und Statistik an der Ludwigs-Maximilians-Universität in München. 1944 wurde er im 2. Weltkrieg zum Militärdienst bei der schweren Artillerie eingezogen. Das Kriegsende und die Entlassung aus Gefangenschaft erlebte er 1945 in Tschechien. Er kehrte ohne Verletzungen heim und konnte dann bald sein Studium an der Münchener Universität fortsetzen. Im Jahre 1947 erwarb er den Grad eines Diplommathematikers, im Jahre 1949 wurde er dann zum Dr. rer. nat. bei Prof. Oscar Andersen sen. promoviert. Ab Anfang 1948 war er als wissenschaftlicher Mitarbeiter, danach als Referent im Statistischen Landesamt Bayern angestellt. Mit dem Buch Moderne Methoden in der Agrarstatistik habilitierte er sich in München im Jahre 1955. Seine Habilitationsschrift fand hohe Anerkennung und wurde in mehrere Fremdsprachen übersetzt. Seinem Doktorvater Oscar Andersen sen. blieb Heinrich Strecker bis zu dessen Tod freundschaftlich verbunden.

Nacheinander erreichten Dr. Strecker Rufe an die Wirtschaftshochschule Mannheim und die Eberhard-Karls-Universität Tübingen. Nach Tübingen wurde er 1959 zum o. Prof. für Statistik und Mathematik für Wirtschaftswissenschaftler berufen; danach stand er bis zu seiner Emeritierung auch der Abteilung für Wirtschafts- und Sozialstatistik vor. In den folgenden Jahren erreichten ihn vier weitere, ehrenvolle Rufe,

R. Wiegert ( $ه)$

72116 Mössingen, Deutschland 
denen er jedoch im Interesse der Kontinuität der Aufgaben in Forschung, Herausgebertätigkeit und Lehre an seinem Tübinger Lehrstuhl und Institut nicht folgte. Im Jahre 1962 heirateten Frau Dr. Rosemarie Bassenge und Professor Dr. Heinrich Strecker in München und begannen dort ihren langen harmonischen und glücklichen Lebensweg. Von ihrem Wohnsitz in Tübingen aus unternahmen sie viele Reisen, die sie zu interessanten Menschen und Ländern führten, u.a. in die USA nach Washington, wo sich die Gelegenheit ergab, die Arbeit des Bureau of the Census und die Kollegen vor Ort kennen zu lernen. Ein langjähriger Austausch knüpfte sich daran an. Eine weitere große Reise führte die Streckers nach Japan, wo sie als Hausgäste bei einem renommierten Kollegen eingeladen wurden und dadurch Einblicke in Usancen und Lebensgewohnheiten des japanischen Privatlebens und natürlich auch der Besonderheiten der japanischen amtlichen Statistik gewinnen konnten. In summa darf man sagen, Streckers reisten gern und weit, so gewannen sie damit Bild und Inhalt der menschlichen Realität und ihrer Konditionen angenähert global. Ihre Reisen waren das Eine, doch ergänzt wurde dies durch Urbanität und Gastfreundschaft den ausländischen Gastwissenschaftlern gegenüber. Dies schuf eine besondere Atmosphäre, welche dem wissenschaftlichen Austausch und der Kollegialität spezifisch zugute kam.

Eine besondere Form Streckerscher Liebe zur Wissenschaft und Kunst zeigte sich in seinen Kenntnissen der Archäologie und der Kunstgeschichte. Er besuchte die großen Münchner Kunstsammlungen, hörte Vorlesungen über die Geschichte der Kunst und ließ sich auf viele Bildungsmöglichkeiten ein, die in besonderer Weise durch München und seine Schätze angeboten waren. Er nutzte sie und wurde so ein Connaisseur von Malerei und Architektur. Er war damit einerseits strenger Wissenschaftler, der in seiner Rigorosität kompromisslos war und der sich andererseits der Kunst und deren Geschichte in besonderer Weise verbunden fühlte. Man gewinnt durch eine solche Geneigtheit an Menschlichkeit und Tiefe von Anschauungen und Einsichten.

Eine weitere Ehrung seiner wissenschaftlichen Arbeit und deren Bedeutung für die Praxis vom Jahre 1971 an wurde ihm durch die Verleihung des Verdienstordens der Bundesrepublik Deutschland zuteil, international bekräftigt durch die Ernennung zum Officier de l'Ordre Leopold II des Königreiches Belgien.

Dieser Nachruf darf nicht enden, bevor die an Heinrich Strecker verliehene Ehrenmitgliedschaft in der Deutschen Statistischen Gesellschaft genannt wurde. Diese Ernennung fand durch ein einstimmiges Votum auf der Jahreshauptversammlung der Gesellschaft im Jahre 1997 statt. Sie war eine verdiente Würdigung der Streckerschen Arbeiten in der Gesellschaft und für sie. Zu seinen Aktivitäten gehörte auch seine Initiative zur Gründung eines Ausschusses für die Qualität statistischer Erhebungen. Dieser Ausschuss ist sowohl bei den Tagungen der Gesellschaft weiterhin präsent als auch jährlich im Herbst beim Statistischen Bundesamt auf einer besonderen Veranstaltung. Herr Strecker war nach der Gründung des Ausschusses für längere Zeit der 1. Vorsitzende.

Professor Strecker wurde am Ende des Sommersemesters 1988 emeritiert. Das Ehepaar Strecker löste seine Tübinger Wohnung auf und übersiedelte in sein Haus nach Starnberg. Dort konnte der Emeritus Strecker noch 25 Jahre mit seiner Frau in der Nähe des sehr geliebten Münchens leben.

Heinrich Strecker verstarb am 17. September 2013 in Starnberg und fand auch dort seine letzte Ruhe. 